\title{
Acute Clostridium Difficile Infection in two Polish Pregnant Women with Premature Rupture of Membranes
}

\author{
Joanna Skręt- Magierło ${ }^{1,2 *}$, Edyta Barnaś ${ }^{2}$, Renata Raś ${ }^{1}$, Krzysztof Gutkowski ${ }^{3}$, Leszek Juszczyk ${ }^{4}$, Alex van Belkum $^{5}$, Hanna Pituch $^{6}$ and \\ Andrzej Skręt ${ }^{1}$ \\ ${ }^{1}$ Clinical Department of Obstetrics and Gynecology, Rzeszów State Hospital, Rzeszów, Poland \\ ${ }^{2}$ Institute of Obstetrics and Emergency Medicine, University of Rzeszów, Poland \\ ${ }^{3}$ Clinical Department of Gastroenterology and Hepatology, Rzeszów State Hospital, Rzeszów, Poland \\ ${ }^{4}$ Obstetrics and Gynecology Hospital „Ujastek”, Crakow, Poland \\ ${ }^{5}$ bioMérieux R\&D Microbiology, La Balme Les Grottes, France \\ ${ }^{6}$ Department of Medical Microbiology, Medical University of Warsaw, Poland
}

\begin{abstract}
We present two cases of acute Clostridium difficile infection (CDI) during pregnancy in Poland. Both patients were hospitalized due to premature rupture of membranes at 15 and 28 weeks of gestation and had antibiotic prophylaxy and therapy. The first patient was discharged on day 18 after the start of the infection but the second patient died after 4 days of hospitalization. Our data and a literature review show that extensive antibiotic usage in such patients may predispose to CDI despite a current lack of detailed studies.BV and health state will provide the depth insight in the etiology of BV.
\end{abstract}

\section{Introduction}

Clostridium difficile are Gram-positive, anaerobic, spore-forming and motile rods. The bacterium was discovered in 1935 but no related diseases were reported in humans until the 70 s of the previous century [1]. C. difficile can be found in the intestines of approximately $2-5 \%$ of adults, $50-60 \%$ of infants and $20-40 \%$ of hospitalized patients. A rapid increase in C. difficile infection (CDI) incidence has been observed in recent years. A growing number of acute infections, associated with elevated complication and mortality rates (even up to $25 \%$, especially among the elderly and seriously ill), has been reported [2,3]. C. difficile produces various virulence factors including toxin $A$, an enterotoxin, and toxin $B$, a potent cytotoxin. During invasion, these toxins damage the intestinal mucosa with consequent necrosis, ulceration, fluid secretion and inflammation. In 2005, an even more virulent strain, i.e. C. difficile-NAP1/BI/027/III (North American PFGE type I/ restriction endonuclease analysis $\mathrm{BI} / \mathrm{PCR}$-ribotype 027, toxin type III), was described. It showed fluoroquinolone resistance, produced an additional binary toxin, and was associated with more severe disease and higher mortality [4-8].

$\mathrm{CDI}$ is characterized by diarrhea or toxic megacolon without a clear cause, coexisting with at least one of the following criteria: i) presence of toxin A and/or B in stool or culture-positive stool, ii) pseudomembranous enterocolitis diagnosed by endoscopy or alternative invasive procedures and/or iii) histopathology [9,10]. Acute CDI is diagnosed if over the course of 30 days since symptom manifestation the following occurred in a patient with the above mentioned symptoms: i) admission to hospital due to community-acquired CDI, ii) admission to ICU due to CDI-related complications, iii) colectomy, iv) death. Treatment is based on cessation of the inciting antibiotics which may suffice in mild infections. Metronidazole or vancomycin (per os) are the two primary antibiotics in the treatment of CDI [11-13].

CDI has been estimated to be the cause of antibiotic-associated diarrhea $(A A D)$ in $15-25 \%$ of the affected individuals $[9,10]$. CDI incidence among hospitalized patients depends on antibiotic use and ranges between 1-10/1000 cases. A significant increase in CDI incidence among hospitalized patients was noted between 1999-2005 in Europe and the USA. In the USA, the incidence increased by $100 \%$ as compared to earlier years [14]. Data from 14 European countries indicate growing
CDI mortality rates, from 2\% in France to $42 \%$ in Great Britain. In 2007, the trend persisted in all countries but England, where a slight decrease in the mortality was observed [15]. The mean incidence of CDI in 27 Polish hospitals was 8.2 per 10,000 patients days as reported in the EUCLID study in 2012. Epidemic spread of fluoroquinolone-resistant C. difficile strains and the role of hospital settings in the development of infections have been documented $[11,16,17]$.

CDI in pregnancy remains rare. According to the Morbidity and Mortality Weekly Report on CDI, pregnant women and parturients are believed to be low-risk populations [18]. The incidence based on data from 1998-2006 was estimated at 0.4-0.7 in 10,000 parturients, mainly including women after cesarean section [19]. Epidemiologic studies estimate the incidence at 7.5 in 1,000 women among parturients [20].

The first description of CDI in pregnancy dated 1985. The literature reports only single cases of the infection, mostly due to broad-spectrum antibiotic therapy, for example prophylactic measures during a cesarean section, premature rupture of membranes and amniotic fluid leak, or group B streptococcus (GBS) infections [21]. Secondary prevention in case of an infection is limited to maintaining proper hygiene.

\section{Case 1}

A 32-year-old woman (para 3, 15 g.w.) presented to the hospital at 12 weeks of gestation due to cervical incompetence. The McDonalds's cerclage was performed. No concomitant diseases were reported. Obstetric history revealed the following: first pregnancy - in 2010,

*Corresponding author: Joanna Skręt- Magierło, Clinical Department of Obstetrics and Gynecology, Rzeszów State Hospital, Rzeszów, Poland, Tel: 4817 87210 00; E-mail: joannaskret@wp.pl

Received June 15, 2015; Accepted August 18, 2015; Published August 21, 2015

Citation: Skręt- Magierło J, Barnaś E, Raś R, Gutkowski K, Juszczyk L, et al. (2015) Acute Clostridium Difficile Infection in two Polish Pregnant Women with Premature Rupture of Membranes. J Medical Microbiol Diagnosis S5:003. doi:10.4172/2161-0703.S5-003

Copyright: (C) 2015 Skręt- Magierło J, et al. This is an open-access article distributed under the terms of the Creative Commons Attribution License, which permits unrestricted use, distribution, and reproduction in any medium, provided the original author and source are credited. 
cesarean section performed at 39 weeks of gestation due to a threat of fetal asphyxia (live newborn - female, birth weight: 2660 grams); second pregnancy - in 2012, cesarean section performed at 26 weeks of gestation due to premature amniotic fluid leak (live newborn - female, birth weight: 850 grams, survived 16 days).

The current third pregnancy required hospitalization at 12 weeks of gestation due to cervical incompetence and at 15 weeks of gestation due to amniotic fluid leak. Vaginal culture was negative. Antibiotic therapy (Cefuroximum 1.5 g, i.v.) was initiated. Diarrhea started on day 9 since amniotic fluid leak ( $>6$ loose stools per day). Stool culture was performed and EIA revealed the presence of glutamate dehydrogenase, after which the presence of toxins was confirmed (BioMerieux VIDAS: C. difficile, toxin A and B: 5.04). Metronidazole $(3 \times 500 \mathrm{mg})$ and Lacidofil (Lactobacillus acidophilus, Lactobacillus rhamnosus) $(3 \times 2$ per os $)$ were initiated. Fetal demise and spontaneous abortion occurred on day 14 since the amniotic fluid leak. The cervical cerclage was removed and a dead male fetus (weight 580 grams), with signs of infection, was instrumentally evacuated. In the afternoon of the same day, the patient was febrile. On the next day the patient was transferred to ICU due to symptoms of anaphylactic shock: pressure 70/40, tachycardia $130 \mathrm{bpm}$, anuria, hypoalbuminemia (1 g/dL). Antibiotic therapy was initiated: Metronidazole $(3 \times 500 \mathrm{mg}$ i.v. $)$, Vancomycin $(3 \times 500 \mathrm{mg}$ i.v.), Ceftazidimum $(3 \times 1 \mathrm{~g}$ iv), Lacidofil (Lactobacillus helveticus, Lactobacillus rhamnosus) $(3 \times 1 \mathrm{~g}$ pre os), and catecholamine infusion. Central venous pressure(CVP) was used to guide fluid therapy. Additional tests revealed indicators of a septic shock (heart rate $>120 \mathrm{u} / \mathrm{min}$; leukocytosis $29.4,19.1 \times 10^{3} / \mathrm{ul}$ ). Diarrhea persists during the course of hospitalization but the second test was negative for $C$. difficile. The patient became stable and symptom-free. Levonor was discontinued. Ultrasound was performed due to persistent generalized edema and revealed accumulating fluid in the abdominal and pleural cavity (laboratory findings confirmed hypoalbuminemia $<2.5 \mathrm{~g} / \mathrm{dL}$ ). On day 7 of ICU stay, single loose stools were observed but the toxin test result was doubtful. The patient became febrile twice $\left(39.0^{\circ} \mathrm{C}\right)$. On day 12 , the blood test came back positive for methicillinresistant coagulase-negative Staphylococcus hemolitycus (MRCNS) and Ciprofloxacine was initiated $(2 \times 400 \mathrm{mg}$ iv). Additionally, albumin infusion and parenteral nutrition were administered. Significant amounts of fluid were evacuated from both pleural cavities on several occasions but the culture results remained negative. Stool tests for $C$. difficile on day 16 of the ICU stay were negative and the patient was transferred to a gastroenterology unit while in a satisfactory condition. Antibiotic therapy was stopped but Lacidofil was continued $(3 \times 1 \mathrm{~g}$ per os). Furosemidum was administered due to fluid in the pleural cavity, followed by thoracentesis - $1000 \mathrm{ml}$ of pleural fluid were evacuated. The patient was discharged on day 6 of hospitalization at the gastroenterology unit in good overall condition.

\section{Case 2}

A 34-year-old primipara was admitted to the hospital at 28 weeks of gestation due to premature amniotic fluid leak for 5 days. Medical history revealed endometriosis, pituitary microadenoma, and infertility treatment. The patient was transferred from the community hospital where she had received tocolytics, steroid therapy, and antibiotic treatment: Cefuroxim $(2 \times 1.5$ g i.v. $)$ and Clindamycin $(2 \times 300 \mathrm{mg}$ i.v. $)$.

In the referral hospital, the patient was put on bed rest, received tocolytics, anticoagulants and antibiotic therapy: Cefuroxim $(3 \times$ $1.5 \mathrm{~g}$ i.v.). Additional tests revealed a urinary tract infection and anemia; conservative treatment was initiated. Genital tract culture was performed on day 7 of hospitalization and revealed Ureaplasma urealyticum infection of the cervix. Owing to amniotic fluid leak, broad-spectrum antibiotic was discontinued and narrow-spectrum antibiotic (Azithromycine $1 \times 0.5 \mathrm{~g}$ per os) was administered to target the genital tract pathogen. After 3 days and a neonatologist's consultation, Azithromycine was changed to another target-antibiotic (Clarithromycin $2 \times 0.5 \mathrm{~g}$ i.v.) due to diarrhea onset and a lower number of loose stools was documented. Fetal status upon ultrasound was normal. Additional tests revealed normal C-reactive protein (CRP) and normal urine values. The patient was afebrile.

On day 12 of hospitalization diarrhea intensified and uterine contractility, only temporarily responsive to intensive pharmacological treatment, was observed. On day 13 of hospitalization, emergency cesarean section was performed due to regular uterine contractions and a threat of intrauterine fetal asphyxia. A live preterm female (birth weight $1240 \mathrm{~g}$, height $40 \mathrm{~cm}$, Apgar score of 6/7/7/8 points in 1/3/5/10 minutes of life) was delivered. The neonate was discharged home in good overall condition on day 62 of life.

Approximately $1000 \mathrm{ml}$ of colorless fluid was found in the abdominal cavity during cesarean section. In the postoperative course the following were observed: persistent diarrhea, elevated temperature on the day of surgery and the postoperative day 1, elevated leukocytosis and CRP, low albumin values, as well as positive stool culture for $C$. difficile toxin $\mathrm{A}$ and $\mathrm{B}$. Albumin, fresh frozen plasma (FFP), colloid and electrolyte infusions and parenteral nutrition were used. Antibiotic therapy was discontinued and Metronidazole treatment $(3 \times 500 \mathrm{mg})$ was started but lowering the number of loose stools was impossible. On postoperative day 2, arterial blood pressure values decreased to undetectable levels and tachycardia $(135 \mathrm{bpm})$ was observed. These values normalized after transfusion of $100 \mathrm{ml}$ of colloids and 3 ampoules of sodium bicarbonate.

Owing to the steadily deteriorating condition of the patient, she was transferred on postoperative day 3 to a higher-referential hospital with ICU, where the presence of $C$. difficile toxin A and B was confirmed and Vancomycin therapy was initiated.

Exploratory laparotomy due to suspected intra-abdominal bleeding and fluid evacuation from the pleural cavity were conducted. Catecholamine infusions (Xigris) were used but no improvement was achieved and the patient died on day 4 after transfer due to septic shock and multi-organ failure.

\section{Discussion}

The two presented cases are severe CDI in pregnancy. According to the European Society of Clinical Microbiology and Infectious Diseases classification, the necessity to transfer a patient to the ICU due to CDI classifies as severe complication [12,13]. Rouphaele et al., report similar cases - data from the USA between 2005-2006, where 10 CDI cases in pregnancy or postpartum (up to 4 weeks after delivery) were registered. Only 3 cases had a mild course, whereas the remaining cases were complicated by toxic megacolon colectomy [6], sepsis [3], and disseminated intravascular coagulation - DCI [4]. Maternal and fetal demise were noted in 3 cases ( 1 twin pregnancy). CDI was diagnosed on the basis of stool testing for $C$. difficile using immune-enzymatic methods, verified by PCR. All of the patients had previously received antibiotic therapy and diarrhea was the main disease manifestation, between 3-60 days after treatment discontinuation. Mean time of hospitalization was 15 days (range: 2-80 days) and all patients were transferred to ICU [22]. 
Citation: Skręt- Magierło J, Barnaś E, Raś R, Gutkowski K, Juszczyk L, et al. (2015) Acute Clostridium Difficile Infection in two Polish Pregnant Women with Premature Rupture of Membranes. J Medical Microbiol Diagnosis S5:003. doi:10.4172/2161-0703.S5-003

The above mentioned case reports are consistent with data reported by Garey et al., who identified 24 cases of CDI in pregnancy (between 1966-2007), despite the fact that in 10 cases data were incomplete. They found that $91 \%$ of the affected women had received prophylactic or therapeutic (50\%) antibiotic therapy. Diarrhea manifested in all patients and stool testing was positive for $C$. difficile and A and/or B toxins (ELISA). Pseudomembranous colitis was confirmed by colonoscopy in 4 women [23].

Venogopal et al., in their retrospective analysis demonstrated CDI incidence during the perinatal period to be $0.7 / 1,000$ gravidas between 2003-2007. In these cases, additional REA type analysis (polymorphic restriction sites) was performed apart from standard immuneenzymatic diagnostic tests (Meridian Bioscience). Different variants of toxins were isolated in 7 patients. These authors concluded that cesarean section may constitute a significant risk factor for CDI in the postpartum period (RR: 11.6\%, CI 95\%). Their findings are consistent with ours, as in both cases Metronidazole therapy, with additional Vancomycin in 1 case, was initiated [21].

The analysis of 20 cases of CDI, whose outbreak was noted among pregnant women in Washington within a course of one year, generated interesting results. CDI incidence was estimated at 7.5/1,000 parturients. CDI-related factors included: cesarean section, antibiotic therapy, and PROM. Combination antibiotic therapy was demonstrated to be an independent risk factor for CDI in the multivariate analysis. According to these authors, pregnant women and parturients appear to be another population susceptible to CDI [20].

Their theory has been confirmed by Candiotto et al., who reported a case of a 39-year-old gravida at 29 weeks of gestation, with a competent immune system, classified as 'low-risk for CDI' with no history of hospitalization or antibiotic therapy in the course of 2 years before the study. CDI, confirmed by ELISA, manifested as diarrhea and led to the development of toxic megacolon and sepsis. Metronidazole treatment $(3 \times 500 \mathrm{mg})$ was initiated. CDI-related complications resulted in transfer to an ICU and, as in case of our patients, hydrothorax which appeared in the patient treated with thoracentesis. The patient made full recovery in the course of three weeks [24].

\section{Conclusions}

Prolonged prophylactic or therapeutic antibiotic therapy in pregnant women with premature rupture of membranes may be associated with the risk of drug-induced CDI.

CDI manifests as diarrhea and in each such case toxin detection and C. difficile-specific stool culture ought to be performed immediately. If the test is positive, Metronidazole treatment should be initiated without delay.

\section{References}

1. Kuijper EJ, Van Dissel JT, Wilcox MH (2007) Clostridium difficile: changing epidemiology and new treatment options. Curr Opin Infect Dis 20: 376-383.

2. Wenisch JM, Schmid D, Tucek G, Kuo HW, Allerberger F, et al. (2012) A prospective cohort study on hospital mortality due to Clostridium difficile infection. Infection 40: 479-84.

3. Dawson LF, Valiente E, Wren BW (2009) Clostridium difficile--a continually evolving and problematic pathogen. Infect Genet Evol 9: 1410-7.

4. Kuijper EJ, Coignard B, Tull P (2006) Emergence of Clostridium difficileassociated disease in North America and Europe. Clin Microbiol Infect 12: 2-18.

5. Clements AC, Magalhaes RJ, Tatem AJ, Paterson DL, Riley TV (2010) Clostridium difficile PCR ribotype 027: assessing the risks of further worldwide spread. Lancet Infect Dis 10: 395-404.
6. Joseph R, Demeyer D, Vanrenterghem D, Van den Berg R, Kuijper E, et al. (2005) First isolation of Clostridium difficile PCR ribotype 027, toxinotype III in Belgium. Euro Surveill 10: E051020.4.

7. Cheknis AK, Sambol SP, Davidson DM, Nagaro KJ, Mancini MC et al. (2009) Distribution of Clostridium difficile strains from a North American, European and Australian trial of treatment for C. difficile infections: 2005-2007. Anaerobe 15: 230-3.

8. Goorhuis A, Bakker D, Corver J, Debast SB, Harmanus C, et al. (2008) Emergence of Clostridium difficile infection due to a new hypervirulent strain, polymerase chain reaction ribotype 078. Clin Infect Dis 47: 1162-70.

9. Bartlett JG, Gerding DN (2008) Clinical recognition and diagnosis of Clostridium difficile infection. Clin Infect Dis 46:S12e8.

10. Cohen SH, Gerding DN, Johnson S, Kelly CP, Loo VG, McDonald LC et al. (2010) Society for healthcare Epidemiology of America; Infectious Diseases Society of America. Clinical practice guidelines for Clostridium difficile infection in adults: 2010 update by the society for healthcare epidemiology of America (SHEA) and the infectious diseases society of America (IDSA). Infect Control Hosp Epidemiol 31: 431e55.

11. Martirosian G, Szczesny A, Cohen SH, Silva Jr J (2005) Analysis of Clostridium difficile associated diarrhea among patients hospitalized in tertiary care academic hospital. Diagn Microbiol Infect Dis 52: 153-5.

12. McDonald C, Coignard B, Dubberke E, Song X, Horan T, et al. (2007) Clostridium difficile Surveillance Working Group. Recommendation for surveillance of Clostridium difficile associated diseases. Infect Control Hosp Epidemiol 28: 140-5.

13. Hryniewicz W, Martirosian G, Ozorowski T. (2011) Clostridium difficile infectiondiagnosis, therapy, prophylactic [in Polish]. National Drugs Institute Warsaw.

14. Elixhauser A, Jhung M (2008) Clostridium difficile Diseases in US Hospitals 1993-2005; AHRQ, Centre for Delivery, Organization and Markets, Healthcare Cost and Utilization Project. Nationwide Inpatient Sample.

15. Wiegand PN, Nathwani D, Wilcox MH, Stephens J, Shelbaya A, et al. (2012) Clinical and economic burden of Clostridium difficile infection in Europe: a systematic review of healthcare-facility-acquired infection. J Hosp Infect 81: 1-14.

16. Pituch $\mathrm{H}$, Bakker $\mathrm{D}$, Kuijper $\mathrm{E}$, Obuch-Woszczatyński $\mathrm{P}$, Wultańska $\mathrm{D}$, et al. (2008) First isolation of Clostridium difficile PCR-ribotype 027/toxinotype III in Poland. Pol J Microbiol 57: 267-8.

17. Davies KA, Longshaw CM, Davis GL, Bouza E, Barbut F, et al. (2014) Underdiagnosis of Clostridium difficile across Europe: the European multicentre, prospective, biannual, point-prevalence study of Clostridium difficile infection in hospitalised patients with diarrhoea (EUCLID). Lancet Infect Dis 14: 1208-19.

18. Centers for Diseases Control and Prevention (CDC). (2005) Severe Clostridum difficile associated diseases in populations previously at low risk-four states, 2005. MMWR Morb Mortal Wkly Rep 54: 1201-5.

19. Kuntz JL, Yang M, Cavanaugh J, Saftlas AF, Polgreen PM (2010) Trends in Clostridium difficile infection among peripartum women. Infection Control 31: 532-534.

20. Unger JA, Whimbey E, Gravett MG, Eschenbach DA (2011) The emergence of Clostridium difficile infection among peripartum women: a case-contro study of a C. difficile outbreak on an obstetrical service. Infect Dis Obstet Gynecol 267249

21. Venugopal AA, Gerding DN, Johnson S (2011) Clostridium difficile infection rates and spectrum of disease among peripartum women at one hospital from 2003 to 2007 with molecular typing analysis of recovered Clostridium difficile isolates. Am J Infect Control 39: 206-11.

22. Rouphael NG, O'Donnell JA, Bhatnagar J, Lewis F, Polgreen PM, et al. (2008) Clostridium difficile-associated diarrhea: an emerging threat to pregnant women. Am J Obstet Gynecol 198: 635.e1-6.

23. Garey KW, Jiang ZD, Yadav Y, Mullins B, Wong K, et al. (2008) Peripartum Clostridium difficile infection: case series and review of the literature. Am J Obstet Gynecol 199: 332-7.

24. Candiotto A, Pascoli I, Gritti A, Busato E, Dal Pozzo G (2010) Toxic megacolon complicating a Clostridium difficile infection in a pregnant woman. J Med Microbiol 59: 124-6. 